\title{
Efficient Perovskite Solar Cells by Temperature Control in Single and Mixed Halide Precursor Solutions and Films
}

Devendra Khatiwada ${ }^{1}$, Swaminathan Venkatesan ${ }^{1}$, Nirmal Adhikari ${ }^{1}$, Ashish Dubey ${ }^{1}$, Abu Farzan Mitul ${ }^{1}$, Lal Mohammad ${ }^{1}$, Anastasiia Iefanova ${ }^{1}$, Seth B. Darling ${ }^{2,3}$, and Qiquan Qiao ${ }^{1}$ *

${ }^{1}$ Center for Advanced Photovoltaics, Department of Electrical Engineering and Computer Science, South Dakota State University, Brookings, SD, USA

Tel: 1-605-688-6965, qiquan.qiao@sdstate.edu

${ }^{2}$ Center for Nanoscale Materials, Argonne National Laboratory, 9700 S. Cass Avenue, Argonne, IL 60439

${ }^{3}$ Institute for Molecular Engineering, University of Chicago, 5640 S Ellis Ave, Chicago, IL 60637
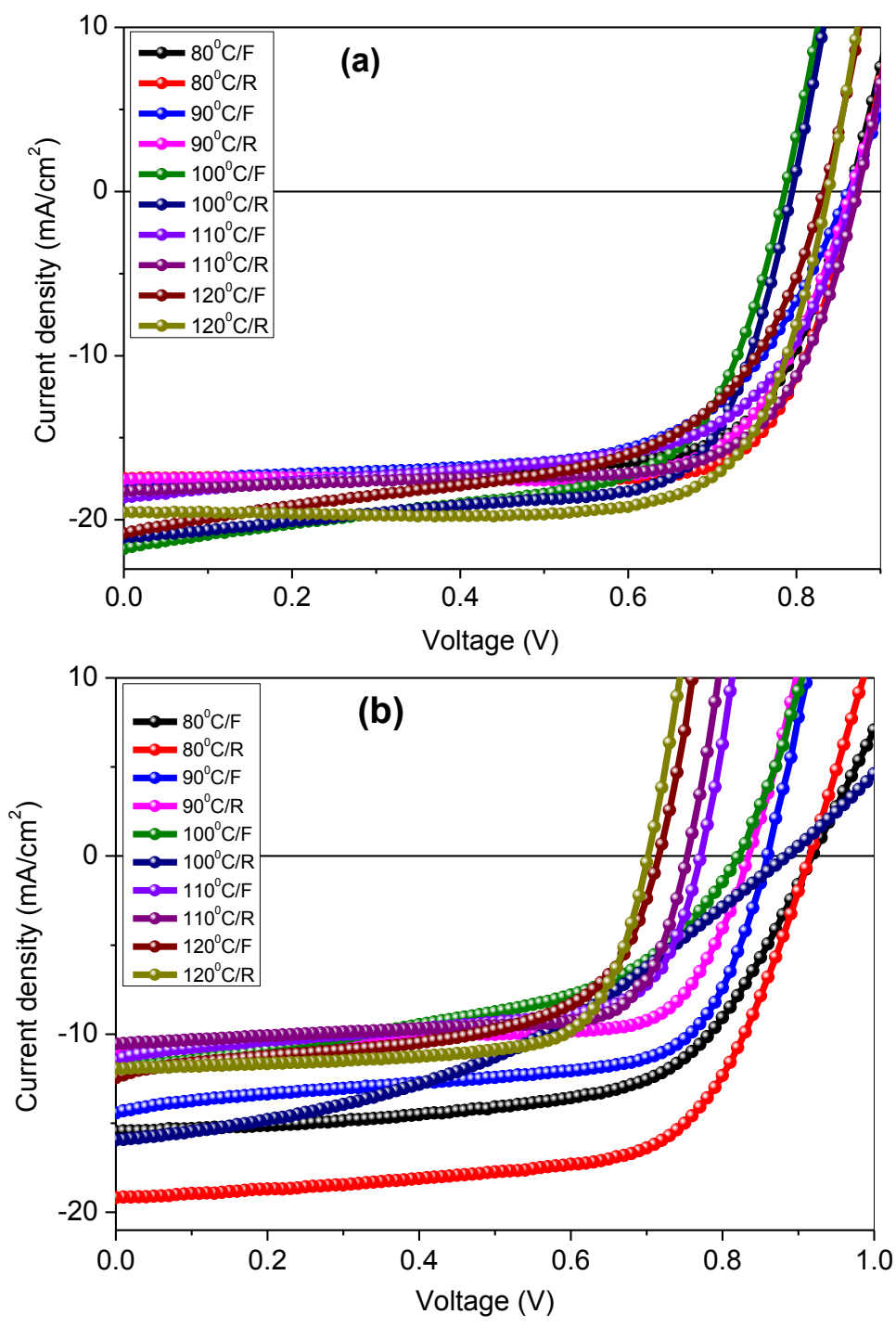
Figure S1. J-V characteristics for (a) singe and (b) mixed halide perovskite solar cells at different film annealing temperature $80{ }^{\circ} \mathrm{C}, 90{ }^{\circ} \mathrm{C}, 100{ }^{\circ} \mathrm{C}, 110{ }^{\circ} \mathrm{C}$ and $120{ }^{\circ} \mathrm{C}$ at both forward (F) and reverse $(\mathrm{R})$ scan. $\mathrm{J}-\mathrm{V}$ characteristics were performed under the same scan rate $(1 \mathrm{~V} / \mathrm{s}$ with step of $10 \mathrm{mV}$ ) for forward and reversed single and mixed halide.

Table S1. Photovoltaic parameters of (a) singe and (b) mixed halide perovskite solar cells at different film annealing temperature $80{ }^{\circ} \mathrm{C}, 90^{\circ} \mathrm{C}, 100^{\circ} \mathrm{C}, 110^{\circ} \mathrm{C}$ and $120^{\circ} \mathrm{C}$ at both forward (F) and reverse $(\mathrm{R})$ scan. $\mathrm{J}-\mathrm{V}$ characteristics were performed under the same scan rate $(1 \mathrm{~V} / \mathrm{s}$ with step of $10 \mathrm{mV}$ ) for forward and reversed single and mixed halide.

\begin{tabular}{|c|c|c|c|c|c|}
\hline \multirow{11}{*}{$\begin{array}{c}\text { Single } \\
\text { halide } \\
\text { (without } \\
\mathrm{PbCl}_{2} \text { ) }\end{array}$} & $\begin{array}{l}\text { Annealing } \\
\text { temperature }\end{array}$ & $\mathrm{V}_{\mathrm{oc}}($ Volt $)$ & $\mathrm{J}_{\mathrm{sc}}\left(\mathrm{mA} / \mathrm{cm}^{2}\right)$ & $\begin{array}{l}\text { Fill factor } \\
\text { (FF) }\end{array}$ & Efficiency \\
\hline & $80{ }^{0} \mathrm{C} / \mathrm{F}$ & $0.86 \pm 0.04$ & $18.09 \pm 0.20$ & 0.68 & $10.71 \pm 0.10$ \\
\hline & $80^{0} \mathrm{C} / \mathrm{R}$ & $0.88 \pm 0.03$ & $17.56 \pm 0.10$ & 0.76 & $11.74 \pm 0.12$ \\
\hline & $90^{0} \mathrm{C} / \mathrm{F}$ & $0.87 \pm 0.01$ & $17.93 \pm 0.10$ & 0.72 & $9.59 \pm 0.12$ \\
\hline & $90{ }^{0} \mathrm{C} / \mathrm{R}$ & $0.79 \pm 0.02$ & $17.61 \pm 0.11$ & 0.62 & $11.05 \pm 0.13$ \\
\hline & $100^{0} \mathrm{C} / \mathrm{F}$ & $0.79 \pm 0.03$ & $21.78 \pm 0.10$ & 0.60 & $10.47 \pm 0.11$ \\
\hline & $100{ }^{0} \mathrm{C} / \mathrm{R}$ & $0.80 \pm 0.03$ & $21.08 \pm 0.10$ & 0.67 & $11.32 \pm 0.10$ \\
\hline & $110^{0} \mathrm{C} / \mathrm{F}$ & $0.88 \pm 0.06$ & $18.26 \pm 0.10$ & 0.56 & $10.08 \pm 0.11$ \\
\hline & $110^{0} \mathrm{C} / \mathrm{R}$ & $0.87 \pm 0.05$ & $18.65 \pm 0.11$ & 0.70 & $11.28 \pm 0.11$ \\
\hline & $120^{\circ} \mathrm{C} / \mathrm{F}$ & $0.83 \pm 0.10$ & $20.18 \pm 0.09$ & 0.56 & $9.72 \pm 0.08$ \\
\hline & $120^{\circ} \mathrm{C} / \mathrm{R}$ & $0.84 \pm 0.01$ & $19.79 \pm 0.08$ & 0.73 & $12.23 \pm 0.10$ \\
\hline \multirow{10}{*}{$\begin{array}{l}\text { Mixed } \\
\text { halide } \\
\text { (with } \\
\mathrm{PbCl}_{2} \text { ) }\end{array}$} & $80^{0} \mathrm{C} / \mathrm{F}$ & $0.92 \pm 0.05$ & $15.46 \pm 0.06$ & 0.61 & $8.97 \pm 0.10$ \\
\hline & $80^{\circ} \mathrm{C} / \mathrm{R}$ & $0.92 \pm 0.06$ & $15.46 \pm 0.12$ & 0.61 & $8.97 \pm 0.12$ \\
\hline & $90^{0} \mathrm{C} / \mathrm{F}$ & $0.86 \pm 0.07$ & $14.42 \pm 0.10$ & 0.64 & $7.95 \pm 0.10$ \\
\hline & $90^{\circ} \mathrm{C} / \mathrm{R}$ & $0.84 \pm 0.07$ & $10.94 \pm 0.10$ & 0.70 & $6.47 \pm 0.16$ \\
\hline & $100^{\circ} \mathrm{C} / \mathrm{F}$ & $0.82 \pm 0.04$ & $11.93 \pm 0.11$ & 0.47 & $4.60 \pm 0.10$ \\
\hline & $100^{\circ} \mathrm{C} / \mathrm{R}$ & $0.89 \pm 0.04$ & $15.94 \pm 0.15$ & 0.40 & $5.69 \pm 0.18$ \\
\hline & $110^{0} \mathrm{C} / \mathrm{F}$ & $0.77 \pm 0.08$ & $11.46 \pm 0.16$ & 0.62 & $5.48 \pm 0.12$ \\
\hline & $110^{\circ} \mathrm{C} / \mathrm{R}$ & $0.76 \pm 0.05$ & $10.56 \pm 0.16$ & 0.71 & $5.73 \pm 0.15$ \\
\hline & $120^{\circ} \mathrm{C} / \mathrm{F}$ & $0.72 \pm 0.02$ & $12.46 \pm 0.12$ & 0.56 & $5.07 \pm 0.10$ \\
\hline & $120^{\circ} \mathrm{C} / \mathrm{R}$ & $0.71 \pm 0.08$ & $11.93 \pm 0.17$ & 0.69 & $5.92 \pm 0.14$ \\
\hline
\end{tabular}




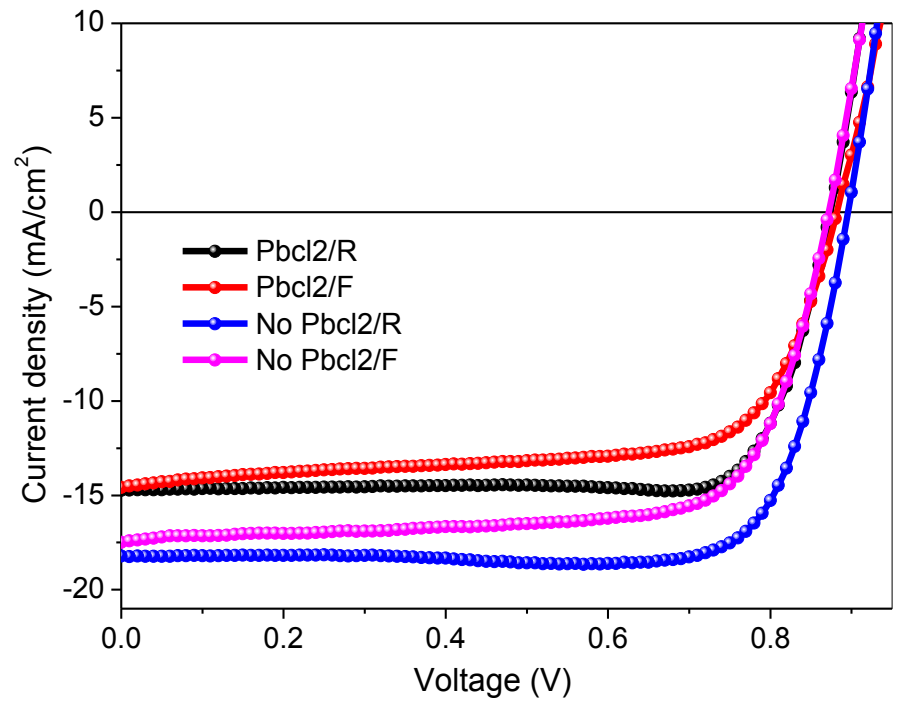

Figure S2. J-V characteristics of perovskite solar cells processed with and without $\mathrm{PbCl}_{2}$ using heated solution $\left(>150{ }^{\circ} \mathrm{C}\right)$ at both forward $(\mathrm{F})$ and reverse $(\mathrm{R})$ scan. The annealing temperature for the following films is $80{ }^{\circ} \mathrm{C}$. $\mathrm{J}-\mathrm{V}$ characteristics were performed under same scan rate $(1 \mathrm{~V} / \mathrm{s}$ with step of $10 \mathrm{mV}$ ) for forward and reversed single and mixed halide.

Table S2. Photovoltaic parameters of (a) singe and (b) mixed halide perovskite solar cells processed with and without $\mathrm{PbCl}_{2}$ using heated solution $\left(150{ }^{\circ} \mathrm{C}\right)$. The annealing temperature for the following films is $80^{\circ} \mathrm{C}$ at both forward (F) and reverse (R) scan. $\mathrm{J}-\mathrm{V}$ characteristics were performed under the same scan rate $(1 \mathrm{~V} / \mathrm{s}$ with step of $10 \mathrm{mV})$ for forward and reversed single and mixed halide.

\begin{tabular}{|c|c|c|c|c|c|}
\hline & $\begin{array}{c}\text { Annealing } \\
\text { temperature }\end{array}$ & $\mathrm{V}_{\mathrm{oc}}(\mathrm{Volt})$ & $\mathrm{J}_{\mathrm{sc}}\left(\mathrm{mA} / \mathrm{cm}^{2}\right)$ & $\begin{array}{c}\text { Fill factor } \\
(\mathrm{FF})\end{array}$ & Efficiency \\
\hline $\begin{array}{c}\text { Single halide } \\
\left.\text { (Without } \mathrm{PbCl}_{2}\right)\end{array}$ & $80^{0} \mathrm{C} / \mathrm{F}$ & $0.87 \pm 0.06$ & $17.48 \pm 0.02$ & 0.72 & $11.01 \pm 0.02$ \\
\cline { 2 - 6 } & $80{ }^{0} \mathrm{C} / \mathrm{R}$ & $0.90 \pm 0.01$ & $18.65 \pm 0.01$ & 0.78 & $13.14 \pm 0.01$ \\
\hline $\begin{array}{c}\text { Mixed halide }(\mathrm{With} \\
\left.\mathrm{PbCl}_{2}\right)\end{array}$ & $80^{0} \mathrm{C} / \mathrm{F}$ & $0.88 \pm 0.03$ & $14.56 \pm 0.01$ & 0.68 & $8.79 \pm 0.03$ \\
\cline { 2 - 6 } & $80^{0} \mathrm{C} / \mathrm{R}$ & $0.88 \pm 0.02$ & $14.77 \pm 0.02$ & 0.81 & $10.56 \pm 0.02$ \\
\hline
\end{tabular}



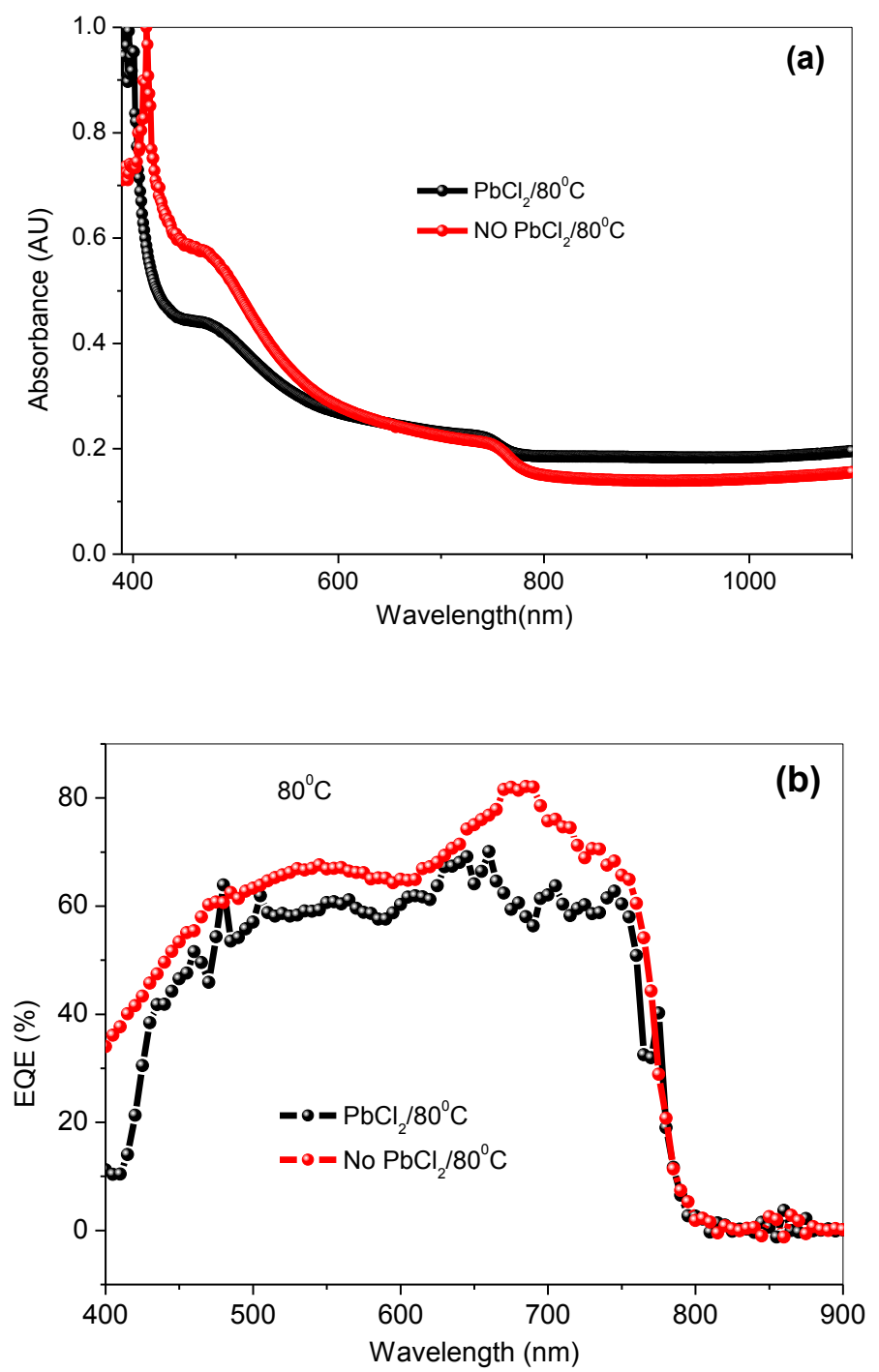

Figure S3. (a) UV-vis absorbance and (b) EQE for perovskite solar cells fabricated from heated solution $\left(150{ }^{\circ} \mathrm{C}\right)$. The annealing temperature for the following films is $80^{\circ} \mathrm{C}$. 


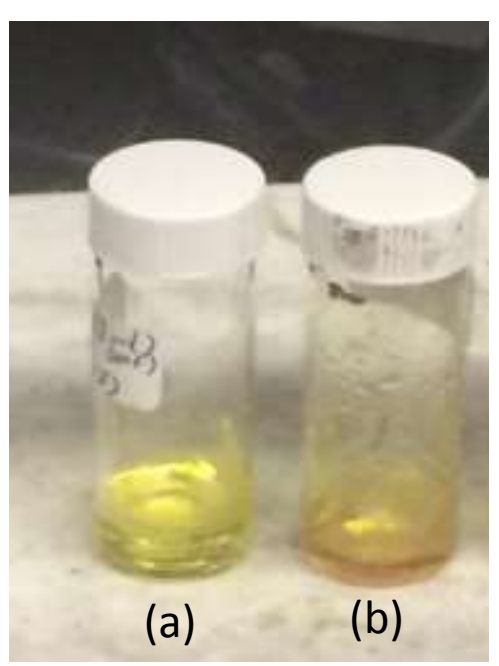

Figure S4. Photo of heated solution at (a) $60{ }^{\circ} \mathrm{C}$ and (b) $150{ }^{\circ} \mathrm{C}$. Color changes from yellow to light brown after heated from $60{ }^{\circ} \mathrm{C}$ to $150{ }^{\circ} \mathrm{C}$. 